\title{
Improved dynamical modelling of the Arches cluster
}

\author{
Joowon Lee ${ }^{1}$ and Sungsoo S. Kim ${ }^{1,2}$ \\ ${ }^{1}$ School of Space Research, Kyung Hee University, \\ Yongin, Gyeonggi, 446-701, Korea \\ email: jwlee9033@khu.ac.kr \\ ${ }^{2}$ Department of Astronomy and Space Science, Kyung Hee University, \\ Yongin, Gyeonggi, 446-701, Korea
}

\begin{abstract}
Recently, Clarkson et al. (2012) measured the intrinsic velocity dispersion of the Arches cluster, a young and massive star cluster in the Galactic center. Using the observed velocity dispersion profile and the surface brightness profile of Espinoza et al. (2009), they estimate the cluster's present-day mass to be $\sim 1.5 \times 10^{4} \mathrm{M}_{\odot}$ by fitting an isothermal King model. In this study, we trace the best-fit initial mass for the Arches cluster using the same observed data set and also the anisotropic Fokker-Planck calculations for the dynamical evolution.
\end{abstract}

Keywords. Galaxy: globular clusters: individual(Arches)-Galaxy: kinematics and dynamics

\section{Introduction}

The Arches cluster is a young, massive and dense cluster near the Galactic center. It is located $\sim 26 \mathrm{pc}$ in projection from the Galactic center. Clarkson et al. (2012) detected the intrinsic velocity dispersion of the Arches cluster. By comparing the model with their velocity dispersion data sets, the present-day mass for the Arches cluster was traced. In this paper, we study the dynamical evolution of the Arches cluster using Fokker-Planck calculations and trace the initial mass range of the Arches cluster, which simultaneously represent the observations of velocity dispersion (Clarkson et al. 2012), surface density (Espinoza et al. 2009) profiles, and the mass function (Kim et al. 2006).

\section{Initial conditions}

For initial conditions of Fokker-Planck models, we adopt a King model. To trace the initial mass range of the Arches cluster, we tried various parameters for King models. The concentration parameter, $W_{0}$, was tried at values 1,4 , and 8 . As galactocentric radius, $R_{g}$, we use $30,40,50$ and 70 . The tidal Radius, $R_{t}$, is constrained by $R_{g}$ and the initial mass of the cluster. For the lower and upper mass boundaries, we used 0.1 and $150 \mathrm{M}_{\odot}$, and we adopted the Kroupa mass function. For all of the parameters, we surveyed an initial mass range of the cluster for each model, $(1-7) \times 10^{4} \mathrm{M}_{\odot}$.

\section{Results}

We compare the observational data with our Fokker-Planck results at $2 \mathrm{Myr}$ and calculate the figure of merit of $\chi_{v}^{2}, \chi_{\Sigma}^{2}$ and $\chi_{m f}^{2}$ (for velocity dispersion, surface density and mass function). Each figure of merit is

$$
\chi_{v, \Sigma, m f}^{2}=\sum_{i=1}^{N} \frac{[\text { data }- \text { model }]_{i}^{2}}{\Delta_{\text {data }, i}^{2}}
$$


Table 1. FP models with different initial conditions

\begin{tabular}{|ccc|ccc|ccc|}
\hline Model & $W_{0}$ & $R_{g}$ & Model & $W_{0}$ & $R_{g}$ & Model & $W_{0}$ & $R_{g}$ \\
130 & 1 & 30 & 430 & 4 & 30 & 830 & 8 & 30 \\
140 & 1 & 40 & 440 & 4 & 40 & 840 & 8 & 40 \\
150 & 1 & 50 & 450 & 4 & 50 & 850 & 8 & 50 \\
170 & 1 & 70 & 470 & 4 & 70 & 870 & 8 & 70 \\
\hline
\end{tabular}
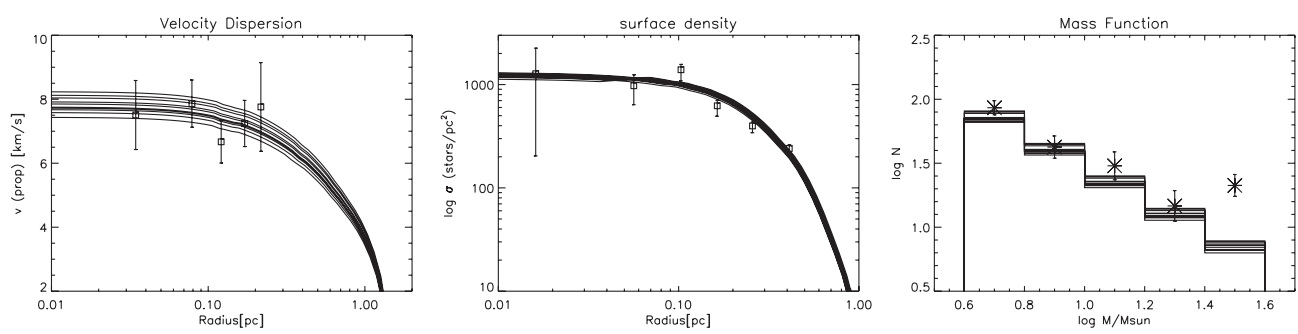

Figure 1. The velocity dispersion, Surface density and Mass function profiles.

Here, $\mathrm{N}$ is the number of data points and $\Delta^{2}$ represents the squared errors on each data point. Then the full figure of merit is $\chi_{\text {full }}^{2}=\chi_{v}^{2}+\chi_{\Sigma}^{2}+\chi_{m f}^{2}$.

To find the plausible initial mass range for the Arches cluster, we choose the model which has the smallest value of the full figure of merit varying the $M_{\text {init }}$. We calculate the acceptable initial mass range having $\chi_{\text {full }}^{2}$ within $95.4 \%$ significance (less than 8.02 ) for model 140, and find the initial mass range of the Arches cluster as $M_{\text {init }}=(1.9-2.4) \times 10^{4}$ $\mathrm{M}_{\odot}$.

Figure 1 shows the velocity dispersion (left panel), surface density (middle panel) and mass function profile (right panel) of model 140 within $\Delta \chi_{\text {full }}^{2}<8.02$. Data of velocity dispersion (Clarkson et al. 2012), surface density (Espinoza et al. 2009) and mass function (Kim et al. 2006) are used.

\section{Summary}

Using Fokker-Planck calculation, we compare the results of the calculation with the observations of velocity dispersion, surface density, and mass function. We find that the acceptable initial mass range of the Arches cluster, which has the smallest $\chi_{\text {full }}^{2}$ value in our models, to be $M_{\text {init }}=(1.9-2.4) \times 10^{4} \mathrm{M}_{\odot}$.

\section{References}

Clarkson, W. I., Ghez, A. M., Morris, M. R., et al. 2012, ApJ 751, 132

Espinoza, P. \& Selman, F. J., Melnic, J. 2009, A\& $A$ 501, 563

Kim, S. S., Figer, D. F., Kudritzki, R. P., \& Najarro, F. 2006, ApJ 653, L113

Kim, S. S., Morris, M., \& Lee, H. M. 1999, ApJ 525, 22 